Multidimensional wave packet dynamics within the fluid dynamical formulation of the Schrödinger equation

Bijoy K. DeyAttila AskarH. Rabitz

Citation: The Journal of Chemical Physics 109, 8770 (1998); doi: 10.1063/1.477547

View online: http://dx.doi.org/10.1063/1.477547

View Table of Contents: http://aip.scitation.org/toc/jcp/109/20

Published by the American Institute of Physics

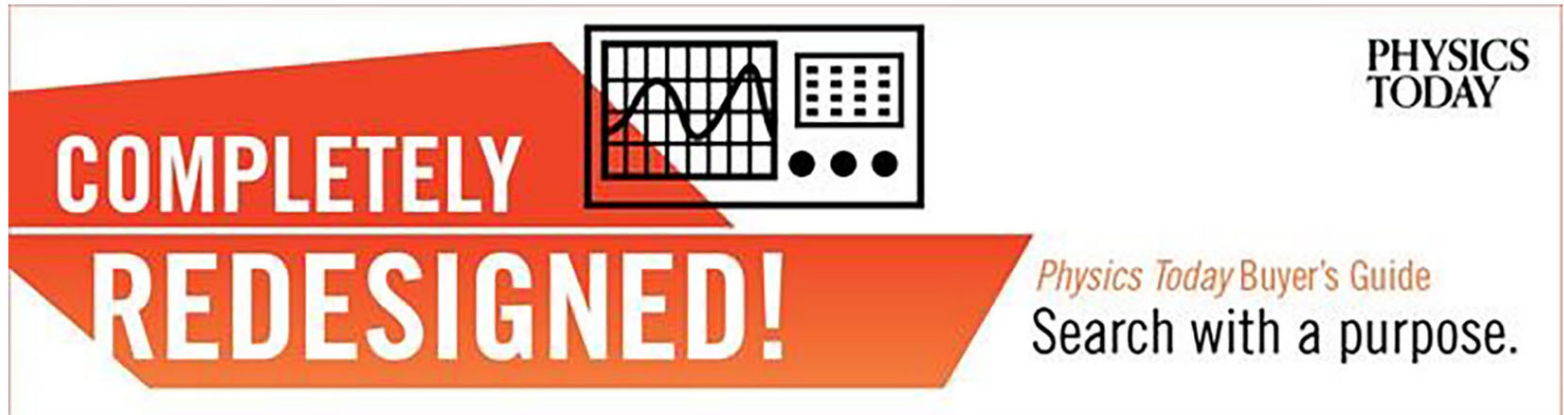




\title{
Multidimensional wave packet dynamics within the fluid dynamical formulation of the Schrödinger equation
}

\author{
Bijoy K. Dey ${ }^{\text {a) }}$ \\ Department of Mathematics, Koc University, Istanbul, Istinye, Turkey and Department of Chemistry, \\ Princeton University, Princeton, New Jersey 08544 \\ Attila Askar \\ Department of Mathematics, Koc University, Istanbul, Istinye, Turkey \\ H. Rabitz \\ Department of Chemistry, Princeton University, Princeton, New Jersey 08544
}

(Received 6 May 1998; accepted 21 August 1998)

\begin{abstract}
This paper explores the quantum fluid dynamical (QFD) representation of the time-dependent Schrödinger equation for the motion of a wave packet in a high dimensional space. A novel alternating direction technique is utilized to single out each of the many dimensions in the QFD equations. This technique is used to solve the continuity equation for the density and the equation for the convection of the flux for the quantum particle. The ability of the present scheme to efficiently and accurately describe the dynamics of a quantum particle is demonstrated in four dimensions where analytical results are known. We also apply the technique to the photodissociation of $\mathrm{NOCl}$ and $\mathrm{NO}_{2}$ where the systems are reduced to two coordinates by freezing the angular variable at its equilibrium value. (C) 1998 American Institute of Physics.
\end{abstract}

[S0021-9606(98)02444-1]

\section{INTRODUCTION}

Time-dependent quantum dynamics of complex systems have gained much attention lately for the ability to attain solutions in a reasonable amount of computational time. In addition, the time-dependent solutions offer an intuitive description of physical processes, especially when coupled with the graphical presentations of the solutions.

Various methods have been proposed for solving the time-dependent Schrödinger equation (TDSE). ${ }^{1-14}$ The purpose of this paper is to demonstrate a novel technique to numerically integrate the TDSE in $N \geqslant 1$ degrees of freedom within an affordable time. We consider the hydrodynamic formulation by Bohm ${ }^{15}$ which replaces the TDSE by two fluid dynamical equations, viz. a continuity equation for the density and an equation of motion for the current density, thus providing an exact classical like analogy to quantum mechanics. Since the derivation of these equations there have been various dynamical applications. ${ }^{16-29}$ The work in Refs. 21-26 utilizes the Eulerian scheme of representing quantum fluid dynamics (QFD) by a nonlinear Schrodinger equation (NLSE) whereas those in Refs. 16-21 are based on the Lagrangian scheme which follow a collection of trajectories of the "fictitious" particles (i.e., the nodes of the irregularly spaced grid with the same fixed mass at all nodes and at all times). The present work demonstrates a new technique to solve the hydrodynamical equations of Bohm by adapting the recently developed Flux Corrected Transport (FCT) algorithm, ${ }^{30}$ which may be used either for the Eulerian or Lagrangian scheme. We show the feasibility of carrying out

${ }^{a)}$ Present address: Department of Chemistry, University of Toronto, Toronto, Ontario M5S 1A1, Canada; electronic mail: bdey@tikva.chem.utoronto.ca quantum dynamics in high dimensions with the new technique.

Section II presents the background of the new alternating direction technique which separates all the coordinates involved in the hydrodynamical equations in many dimensions to yield a set of equations with each containing only one coordinate. Here we utilize Cartesian coordinates, although the extension to other coordinate systems follow similarly. Section III briefly gives the numerical features of the FCT algorithm. Modification of the algorithm is derived in the present context of the quantum fluid dynamical equations which possess an additional quantum potential. Section IV applies the technique to two different problems: (A) Evolution of a four-dimensional wave packet under a harmonic potential with both positive and negative curvatures and (B) photodissociation of $\mathrm{NOCl}$ and $\mathrm{NO}_{2}$. The results for these two problems are discussed separately in this section. Section $\mathrm{V}$ presents future research topics related to this technique and concludes the paper.

\section{ALTERNATING DIRECTION TECHNIQUE FOR QUANTUM HYDRODYNAMICAL EQUATIONS}

The hydrodynamical analogy of quantum mechanics is obtained by expressing the Schrödinger wave function as $\psi$ $=\rho^{1 / 2} e^{i S}$ where $\rho$ and $S$ are real functions. Substitution into the time-dependent Schrödinger equation (TDSE) $i \partial \psi / \partial t$ $=\left(-1 / 2 \nabla^{2}+V\right) \psi$ and separation of the real and imaginary parts leads to two equations (see Ref. 15) viz.

$$
\frac{\partial \rho}{\partial t}+\nabla \cdot(\rho \mathbf{v})=0
$$

and 


$$
\frac{\partial S}{\partial t}+\frac{1}{2} v^{2}+V+V_{q}=0
$$

where $\quad \rho=|\psi|^{2}, \quad \mathbf{v}=\boldsymbol{\nabla} S, \quad V_{q}=-\frac{1}{2} \nabla^{2} \rho^{1 / 2} / \rho^{1 / 2}=$ $-\frac{1}{2}\left[\nabla^{2} \log \rho^{1 / 2}+\left(\nabla \log \rho^{1 / 2}\right)^{2}\right]$ and $V$ is the usual potential. The above equations are for a single particle of unit mass where atomic units are assumed. An extension of the formalism for many-particle systems can readily be made. The present single particle formulation is adequate for the multiparticle case illustrated later as they can be transformed to be consistent with the equation above.

The many facets of these QFD equations have been presented in recent work. ${ }^{18}$ Here we shall work with the following equivalent sets of hydrodynamical equations viz.

$$
\frac{\partial \rho}{\partial t}=-\nabla \cdot(\rho \mathbf{v})
$$

and

$$
\frac{\partial \mathbf{j}}{\partial t}=-\boldsymbol{\nabla} \cdot(j \mathbf{v})-\rho \boldsymbol{\nabla}\left(V+V_{q}\right),
$$

where $\mathbf{j}=\rho \mathbf{v}$ is the current density vector for the particle with density $\rho$ and velocity vector $\mathbf{v}$. Equation (4) can be obtained from Eqs. (1) and (2) after minor rearrangements. The form in Eq. (4) is favorable from a computational viewpoint. The algorithm that is employed is based on the flux conservation and the generic equation is of flux type. In Eq. (4), the last term plays the role of a source and is weighted by the density. This weighting discerns the regions of the space with high and low probability density and has a stabilizing effect on the numerics.

To present an understanding of the alternating direction technique (ADT) for solving the hydrodynamical equations, Eqs. (3) and (4), consider a dynamical system in $n$ spatial coordinates $\left(x_{d} ; d=1,2, \ldots, n\right)$ and with the basis set $\mathbf{e}_{\mathbf{k}}$, i.e.,

$$
\begin{aligned}
\rho(x) & \equiv \rho\left(x_{1}, x_{2}, \ldots, x_{n}\right), \\
\mathbf{j}(x) & \equiv \sum_{k=1}^{n} \mathbf{e}_{\mathbf{k}} j_{k}\left(x_{1}, x_{2}, \ldots, x_{n}\right) .
\end{aligned}
$$

The $n$-dimensional coordinate system is assumed to be orthogonal. Thus, Eqs. (3) and (4) can be written as

$$
\frac{\partial \rho}{\partial t}=-\sum_{k=1}^{n} \frac{\partial}{\partial x_{k}}\left(\rho v_{k}\right)
$$

and

$$
\begin{aligned}
\frac{\partial j_{m}}{\partial t}= & -\frac{\partial}{\partial x_{m}}\left(j_{m} v_{m}\right)-\rho \frac{\partial}{\partial x_{m}}\left(V+V_{q}\right) \\
& -\sum_{k=1, k \neq m}^{n} \frac{\partial}{\partial x_{k}}\left(j_{m} v_{k}\right), \quad m=1,2, \ldots, n .
\end{aligned}
$$

In the alternating direction scheme, Eqs. (5) and (6) are split into $n$ sets of one-dimensional equations, such that each set contains $n+1$ equations. For the $m$ th set $1 \leqslant m \leqslant n$, we have the following $n+1$ equations: $:^{30}$

$$
\begin{aligned}
& \frac{\partial \rho}{\partial t}=-\frac{\partial}{\partial x_{m}}\left(\rho v_{m}\right), \\
& \frac{\partial j_{k}}{\partial t}=-\frac{\partial}{\partial x_{m}}\left(j_{k} v_{m}\right)+S_{k}, \quad k=1,2, \ldots, m, \ldots, n,
\end{aligned}
$$

where $S_{k}$ is the source term associated with the evolution of $j_{k}$, such that

$$
S_{k}=\left\{\begin{array}{l}
-\rho \frac{\partial}{\partial x_{m}}\left(V+V_{q}\right), \quad k=m \\
0, \quad k \neq m .
\end{array}\right.
$$

There are $n$ such sets of equations. Notice that for the $m$ th set of equations, we obtain an intermediate value of density and $n$ intermediate components of the current density vector, all of which are convected by the $m$ th component of the velocity $\left(v_{m}\right)$. The equation for $m$ th component of current has a definite source and the other component sources are zero. The above splitting is such that each set of equations contains only one spatial coordinate, thus reducing the problem to a one space dimensional case and thus making the solution easier.

The numerical steps in the alternating direction scheme go as follows

Step 1: At $t=0, \rho$ and $v_{d}, d=1,2, \ldots, m, \ldots, n$ are given.

Step 2: Compute the quantum potential given by

$$
V_{q}=-\frac{1}{2} \nabla^{2} \log \rho^{1 / 2}-\frac{1}{2}\left(\boldsymbol{\nabla} \log \rho^{1 / 2}\right)^{2} .
$$

Step 3: Construct the first set of equations where velocity is $v_{1}$.

$$
\begin{aligned}
& \frac{\partial \rho}{\partial t}=-\frac{\partial}{\partial x_{1}}\left(\rho v_{1}\right), \\
& \frac{\partial j_{1}}{\partial t}=-\frac{\partial}{\partial x_{1}}\left(j_{1} v_{1}\right)-\rho \frac{\partial}{\partial x_{1}}\left(V+V_{q}\right), \\
& \frac{\partial j_{2}}{\partial t}=-\frac{\partial}{\partial x_{1}}\left(j_{2} v_{1}\right), \\
& \frac{\partial j_{n}}{\partial t}=-\frac{\partial}{\partial x_{1}}\left(j_{n} v_{1}\right) .
\end{aligned}
$$

Step 4: Solve this set of $n+1$ equations to obtain the intermediate values $\rho^{\text {int }}, j_{d}^{\text {int }}, d=1,2, \ldots, n$ at time $t=\delta t$.

Step 5: Replace $\rho^{\mathrm{int}}$ by $\rho$ and $j_{d}^{\mathrm{int}}$ by $j_{d}, d=1,2, \ldots, n$. Then calculate $V_{q}$ from $\rho$ and $v_{d}$ from $v_{d}=j_{d} / \rho, d$ $=1,2, \ldots, n$.

Step 6: Construct the second set of equations which involve velocity $v_{2}$ calculated in step 4 ,

$$
\begin{aligned}
& \frac{\partial \rho}{\partial t}=-\frac{\partial}{\partial x_{2}}\left(\rho v_{2}\right), \\
& \frac{\partial j_{1}}{\partial t}=-\frac{\partial}{\partial x_{2}}\left(j_{1} v_{2}\right), \\
& \frac{\partial j_{2}}{\partial t}=-\frac{\partial}{\partial x_{2}}\left(j_{2} v_{2}\right)-\rho \frac{\partial}{\partial x_{2}}\left(V+V_{q}\right) .
\end{aligned}
$$




$$
\frac{\partial j_{n}}{\partial t}=-\frac{\partial}{\partial x_{2}}\left(j_{n} v_{2}\right) .
$$

Step 7: Solve the second set of equations to obtain another set of intermediate values for $\rho^{\text {int }}$ and $j_{d}^{\text {int }}, d$ $=1,2, \ldots, n$ at time $t=\delta t$.

Step 8: Return to step 4 and construct the next set of equations and solve them to obtain $\rho^{\text {int }}$ and $j_{d}^{\text {int }}, d$ $=1,2, \ldots, n$, until all $n$ sets of equations are solved.

Step 9: The final results for $\rho^{\text {int }}$ and $j_{d}^{\text {int }}, d=1,2, \ldots, n$ obtained after solving the $n$th set of equations are the desired values of $\rho$ and $j_{d}$ at $t=\delta t$.

Step 10: Repeat steps 1-8 over a number of time steps. The resultant equations above can be written in a general form as

$$
\frac{\partial C}{\partial t}=-\frac{\partial}{\partial x}(C v)+\frac{\partial}{\partial x}\left(D_{1}\right)+F \frac{\partial D_{2}}{\partial x}+D_{3},
$$

where $C$ is the quantity to be convected in the $x$ direction with respect to the given velocity $v$. The first term on the right-hand side is associated with the conservation of $C$ and the remaining terms are sources of different structural forms. From the previous equations (7)-(8), we see that for the convection of mass density $(C=\rho)$ there are no source terms (i.e., $D_{1}=D_{2}=D_{3}=0$ ), whereas for the convection of current $(C=j)$ we have $D_{1}=D_{2}=D_{3}=0$ if the velocity is not in the direction of the coordinate and $D_{1}=D_{3}=0, F=-\rho$, $D_{2}=V+V_{q}$ otherwise. In Sec. III, we briefly discuss the FCT numerical method assuming the grid is kept fixed (i.e., the Eulerian formulation).

\section{NUMERICAL SOLUTION OF PROTOTYPE CONVECTION EQUATION}

The simplest three-point discrete formula for Eq. (19) with no source can be written as

$$
C_{i}^{n+1}=C_{i}^{n}-\frac{\delta t}{2 \delta x}\left(C_{i+1}^{n} v_{i+1}^{n}-C_{i-1}^{n} v_{i-1}^{n}\right),
$$

where $i$ is the index for the discrete spatial points and $n$ is that for the time. With a little rearrangement this discretized equation can be rewritten as

$$
\begin{aligned}
C_{i}^{n+1}= & C_{i}^{n}-\frac{1}{2}\left[\epsilon_{i+1 / 2}^{n}\left(C_{i+1}^{n}+C_{i}^{n}\right)-\epsilon_{i-1 / 2}^{n}\left(C_{i}^{n}+C_{i-1}^{n}\right)\right] \\
& +\frac{1}{2}\left[\nu_{i+1 / 2}^{n}\left(C_{i+1}^{n}-C_{i}^{n}\right)-\nu_{i-1 / 2}^{n}\left(C_{i}^{n}-C_{i-1}^{n}\right)\right],
\end{aligned}
$$

where

$$
\epsilon_{i+1 / 2}^{n}=\frac{1}{2} \frac{\delta t}{\delta x}\left(v_{i+1}^{n}+v_{i}^{n}\right), \quad \epsilon_{i-1 / 2}^{n}=\frac{1}{2} \frac{\delta t}{\delta x}\left(v_{i}^{n}+v_{i-1}^{n}\right)
$$

and

$$
\nu_{i+1 / 2}^{n}=\frac{1}{2} \frac{\delta t}{\delta x}\left(v_{i}^{n}-v_{i+1}^{n}\right), \quad \nu_{i-1 / 2}^{n}=\frac{1}{2} \frac{\delta t}{\delta x}\left(v_{i-1}^{n}-v_{i}^{n}\right) .
$$

The indices $i+\frac{1}{2}$ and $i-\frac{1}{2}$ are the right and left interface, respectively, for any point $i$. We see that $\nu$ is associated with the velocity gradient, thus causing diffusion of $C$ in and out of the interface. $\epsilon$ on the other hand, is directly related to the mean velocity and hence leads to the desired convection. The FCT algorithm attempts to minimize the diffusion which otherwise can lead to spurious results in the solution for $C_{i}$. The FCT algorithm also assures that (i) if $C_{i}^{n}$ is positive at the $n$th time step, then it remains so at any advanced time step, i.e., $\left(C_{i}^{n+1}\right)>0$, (ii) $C$ is conserved if there is no source. Writing Eq. (21) as

$$
C_{i}^{n+1}=\alpha_{i}^{n} C_{i-1}^{n}+\beta_{i}^{n} C_{i}^{n}+\gamma_{i}^{n} C_{i+1}^{n}
$$

one obtains the positivity condition that guarantees all $C_{i}$ 's at all times to remain positive as

$$
\alpha_{i}^{n}, \quad \beta_{i}^{n}, \quad \gamma_{i}^{n} \geqslant 0 .
$$

Similarly the conservation of $C$ is obtained from $\sum_{i} C_{i}^{n+1}$ $=\sum_{i} C_{i}^{n}$ as

$$
\alpha_{i+1}^{n}+\beta_{i}^{n}+\gamma_{i-1}^{n}=1 .
$$

The comparison of Eq. (24) with Eq. (20) yields

$$
\begin{aligned}
\alpha_{i}^{n} & =\frac{1}{2} \frac{\delta t}{\delta x} v_{i-1}^{n}, \\
\beta_{i}^{n} & =1, \\
\gamma_{i}^{n} & =-\frac{1}{2} \frac{\delta t}{\delta x} v_{i+1}^{n}
\end{aligned}
$$

and $\alpha_{i+1}^{n}+\beta_{i}^{n}+\gamma_{i-1}^{n}=1$ is identically satisfied.

It is readily concluded that the formula given by Eq. (24) can lead to positivity of $\left(C_{i}^{n+1}\right), \forall i$ only when $v_{i-1} \geqslant 0$ and $v_{i+1}<0, \forall i$, which is a rather stringent condition. On the other hand Eq. (24) guarantees the conservation of $C_{i}$ at any time step. Thus, a more general algorithm can be constructed by relaxing the conditions in Eqs. (27)-(29) as: $\alpha_{i}^{n}$ $\neq \frac{1}{2}(\delta t / \delta x) v_{i-1}^{n}, \beta_{i}^{n} \neq 1$, and $\gamma_{i}^{n} \neq-\frac{1}{2}(\delta t / \delta x) v_{i+1}^{n}$. Equivalently $\left(\epsilon_{i+1 / 2}^{n}\right)$ and $\left(\nu_{i+1 / 2}^{n}\right)$ are relaxed as not to be given by Eq. (22) and Eq. (23), respectively. As a consequence of these flexibilities, the FCT algorithm modifies $\left(\alpha_{i}^{n}\right),\left(\beta_{i}^{n}\right)$, and $\left(\gamma_{i}^{n}\right)$ as

$$
\begin{aligned}
& \alpha_{i}^{n}=\frac{1}{2}\left(\nu_{i-1 / 2}^{n}+\epsilon_{i-1 / 2}^{n}\right) \neq \frac{1}{2} \frac{\delta t}{\delta x} v_{i-1}^{n}, \\
& \beta_{i}^{n}=1-\frac{1}{2}\left(\epsilon_{i+1 / 2}^{n}+\epsilon_{i-1 / 2}^{n}-\nu_{i+1 / 2}^{n}-\nu_{i-1 / 2}^{n}\right) \neq 1, \\
& \gamma_{i}^{n}=\frac{1}{2}\left(\nu_{i+1 / 2}^{n}-\epsilon_{i+1 / 2}^{n}\right) \neq-\frac{1}{2} \frac{\delta t}{\delta x} v_{i+1}^{n},
\end{aligned}
$$

where $\epsilon_{i+1 / 2}^{n}=(\delta t / \delta x) v_{i+1 / 2}^{n}$ and the value of $\nu_{i+1 / 2}$ is chosen based on (i) the positivity of $\left(C_{i}^{n+1}\right) \forall i$ and (ii) the stability of the algorithm.

The conditions of positivity in Eq. (25) with the above choice of $\alpha, \beta$, and $\gamma$ become

$$
1 \geqslant \nu_{i+1 / 2} \geqslant \epsilon_{i+1 / 2} \text {. }
$$

Similarly, the stability condition is derived with the above choice to $\mathrm{be}^{30}$

$$
1 \geqslant \nu_{i+1 / 2} \geqslant\left|\epsilon_{i+1 / 2}\right|^{2}, \quad\left|\epsilon_{i+1 / 2}\right| \leqslant \frac{1}{2} .
$$

It is seen that the stability condition is a factor of 2 less stringent than the positivity condition. In other words, if the 
positivity condition is satisfied, the stability condition automatically is satisfied if $\left|\epsilon_{i+1 / 2}\right| \leqslant \frac{1}{2}$. However, the reverse is not always true.

The following points are to be noted in regard to the positivity and stability conditions: (i) If importance is given to the positivity condition, i.e., if $\nu_{i+1 / 2}^{n}=\epsilon_{i+1 / 2}^{n}$ (for minimum allowed diffusion) and $\left|\epsilon_{i+1 / 2}\right| \leqslant \frac{1}{2}$, then from Eq. (24) we obtain

$$
C_{i}^{n+1}=\alpha_{i}^{n} C_{i-1}^{n}+\beta_{i}^{n} C_{i}^{n}
$$

with

$$
\alpha_{i}^{n}=\epsilon_{i-1 / 2}^{n}
$$

and

$$
\beta_{i}^{n}=1-\epsilon_{i+1 / 2}^{n} .
$$

This is a two-point first-order upwind scheme and leads to strong diffusion which can cause severe nonphysical spreading. (ii) If importance is given to the stability condition, i.e., if $\nu_{i+1 / 2}^{n}=\left|\epsilon_{i+1 / 2}\right|^{2}$ (i.e., minimum allowed diffusion) then monotonicity of the solution is lost by overshooting or by the Gibbs phenomenon. This can cause unphysical ripples, i.e., new maxima or minima in the solution for $C .^{31}$ This is circumvented in the FCT code $^{32-34}$ by incorporating additional antidiffusion into the solution obtained from Eq. (24) as

$$
\begin{aligned}
\left(C_{i}^{n+1}\right)_{\text {new }}= & C_{i}^{n+1}-\mu_{i+1 / 2}^{n}\left(C_{i+1}^{n+1}-C_{i}^{n+1}\right) \\
& +\mu_{i-1 / 2}^{n}\left(C_{i}^{n+1}-C_{i-1}^{n+1}\right) .
\end{aligned}
$$

Here $\left(\mu_{i+1 / 2}^{n}\right)$ are positive antidiffusion coefficients to reduce the strong diffusion. This mode of reducing diffusion can also lead to new maxima or minima in the new solution discussed below.

Suppose the value of $C_{i}^{n+1}$ at grid point $i$ reaches zero $\left(C_{i}^{n+1}=0\right)$ while its neighbors are positive $\left(C_{i+1}^{n+1}>0\right.$; $\left.C_{i-1}^{n+1}>0\right)$. Then substituting the maximum antidiffusion coefficients obtained from Eq. (37) that still guarantees positivity, i.e.,

$$
\mu_{i+1 / 2}^{n} \simeq \frac{1}{2} \nu_{i+1 / 2}^{n}-\frac{1}{2}\left|\epsilon_{i+1 / 2}^{n}\right|,
$$

in Eq. (37) one obtains

$$
\begin{aligned}
\left(C_{i}^{n+1}\right)_{\text {new }}= & -\frac{1}{2}\left(\nu_{i+1 / 2}^{n}-\left|\epsilon_{i+1 / 2}^{n}\right|\right) C_{i+1}^{n+1} \\
& -\frac{1}{2}\left(\nu_{i-1 / 2}^{n}-\left|\epsilon_{i-1 / 2}^{n}\right|\right) C_{i-1}^{n+1} .
\end{aligned}
$$

Thus $\left(C_{i}^{n+1}\right)_{\text {new }}$ is negative since $\nu_{i+1 / 2}^{n} \geqslant\left|\epsilon_{1+1 / 2}^{n}\right|$. Because the negative value of $C_{i}^{n+1}$ (i.e., unphysical the antidiffusion) should be limited, the FCT code modifies the antidiffusive fluxes ${ }^{32-34}$

$$
f_{i+1 / 2}^{\mathrm{ad}}=\mu_{i+1 / 2}^{n}\left(C_{i+1}^{n+1}-C_{i}^{n+1}\right)
$$

by a process called nonlinear flux correction to ensure both positivity and stability. This leads to the equation

$$
\left(C_{i}^{n+1}\right)_{\text {new }}=C_{i}^{n+1}-f_{i+1 / 2}^{c}+f_{i-1 / 2}^{c},
$$

where $f_{i+1 / 2}^{c}$ is the corrected antidiffusive flux given by

$$
\begin{aligned}
& f_{i+1 / 2}^{c}=S \max \left(0, \min \left[S\left(C_{i+2}^{n+1}-C_{i+1}^{n+1}\right),\right.\right. \\
& \left.\left.\left|f_{i+1 / 2}^{\mathrm{ad}}\right|, S\left(C_{i}^{n+1}-C_{i-1}^{n+1}\right)\right]\right)
\end{aligned}
$$

with $S=\operatorname{sign}\left(C_{i+1}^{n+1}-C_{i}^{n+1}\right)$ and $|S|=1$. In all the above equations $\nu_{i+1 / 2}^{n}$ and $\mu_{i+1 / 2}^{n}$ are of the form

$$
\begin{aligned}
& \nu_{i+1 / 2}^{n}=\frac{1}{3}+\frac{2}{3}\left(\epsilon_{i+1 / 2}^{n}\right)^{2}, \\
& \mu_{i+1 / 2}^{n}=\frac{1}{6}-\frac{1}{6}\left(\epsilon_{i+1 / 2}^{n}\right)^{2} .
\end{aligned}
$$

In the above discussions there was no source. However, when sources of different forms are considered, one can simply add it properly before calculating the antidiffusion flux correction.

Following are the necessary steps to obtain $C_{i}^{n+1}$ from $C_{i}^{n}$ (given).

Step 1: Calculate $C_{i}^{n}$ (for $n=1$, it is assumed given) and the velocity $v_{i}^{n}$ at any given time over a discrete grid.

Step 2: Calculate the convected part of the transported quantity, as

$$
C_{i}^{c}=C_{i}^{n}-\frac{1}{2} \epsilon_{i+1 / 2}^{n}\left(C_{i+1}^{n}+C_{i}^{n}\right)+\frac{1}{2} \epsilon_{i-1 / 2}^{n}\left(C_{i}^{n}+C_{i-1}^{n}\right) .
$$

Step 3: Add the different types of sources as

$$
C_{i}^{c s}=C_{i}^{c}+\left(S_{1}\right)_{i} \delta t+\left(S_{2}\right)_{i} \delta t+\left(S_{3}\right)_{i} \delta t,
$$

where $S_{1}, S_{2}$, and $S_{3}$ are the three different types of sources,

$$
S_{1}=-\frac{\partial}{\partial x} D_{1}, \quad S_{2}=F \frac{\partial}{\partial x} D_{2}, \quad S_{3}=D_{3} .
$$

In wave packet dynamics, we have the $S_{2}$ term given as

$$
S_{2}=-C \frac{\partial}{\partial x}\left(V+V_{q}\right), \quad C=\rho
$$

$V_{q}$ in Eq. (48) is given by Eq. (10) where the numerical evaluation of the first and second derivatives has been performed by central finite difference formula. Thus, the discrete form of $\left(S_{2}\right)_{i}$ is

$$
\left(S_{2}\right)_{i}=-C_{i}^{n} \frac{V_{i+1}+\left(V_{q}\right)_{i+1}-V_{i-1}-\left(V_{q}\right)_{i-1}}{2(\delta x)} .
$$

Step 4: Add diffusion to obtain

$$
C_{i}^{c s D}=C_{i}^{c s}+\frac{1}{2} \nu_{i+1 / 2}^{n}\left(C_{i+1}^{n}-C_{i}^{n}\right)-\frac{1}{2} \nu_{i-1 / 2}^{n}\left(C_{i}^{n}-C_{i-1}^{n}\right) .
$$
$C_{i}^{c s}$ as

Step 5: Calculate the uncorrected antidiffusion flux from

$f_{i+1 / 2}^{\mathrm{ad}}=\nu_{i+1 / 2}^{n}\left(C_{i+1}^{c s}-C_{i}^{c s}\right)$.

Step 6: Calculate the corrected antidiffusive flux as

$$
\begin{aligned}
f_{i+1 / 2}^{\mathrm{cor}}= & S_{i+1 / 2} \max \left(0, \min \left[\left|f_{i+1 / 2}^{\mathrm{ad}}\right|, S_{i+1 / 2}\left(C_{i+2}^{c s D}-C_{i+1}^{c s D}\right),\right.\right. \\
& \left.\left.S_{i+1 / 2}\left(C_{i}^{c s D}-C_{i-1}^{c s D}\right)\right]\right),
\end{aligned}
$$

where

$$
S_{i+1 / 2}=\operatorname{sign}\left(C_{i+1}^{c s D}-C_{i}^{c s D}\right) .
$$

Step 7: The final result at $(n+1)$ th step is

$C_{i}^{n+1}=C_{i}^{c s D}-f_{i+1 / 2}^{\mathrm{cor}}+f_{i-1 / 2}^{\mathrm{cor}}$. 


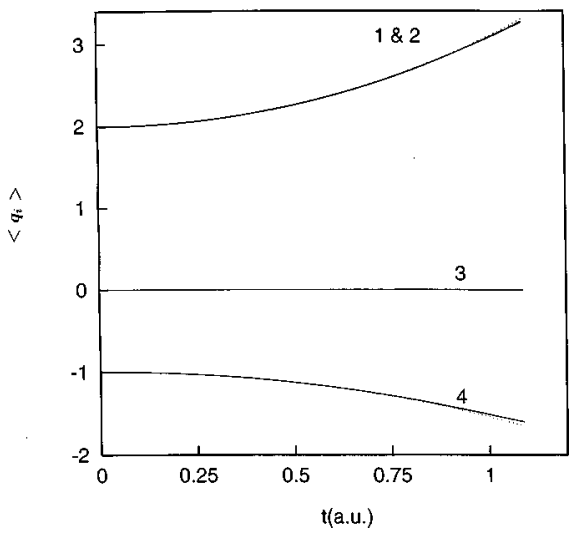

FIG. 1. Mean values $\left(\left\langle q_{i}\right\rangle, i=1,2,3,4\right)$ with respect to time for a $4 \mathrm{D}$ wave packet. The values of $\left\langle q_{1}\right\rangle$ and $\left\langle q_{2}\right\rangle$ increase to the same extent, that of $\left\langle q_{3}\right\rangle$ remains constant whereas $\left\langle q_{4}\right\rangle$ decreases. The corresponding analytical values are shown in dotted lines.

\section{APPLICATIONS}

\section{A. Four-dimensional wave packet propagation}

In this section some test calculations which characterize the motion of a four-dimensional (4D) Gaussian wave packet with a quadratic potential are studied. This can serve as a test of the present computation procedure, since an exact analytical solution ${ }^{17}$ is available. The analytic nature of the problem is not utilized in the numerical procedure and is used only for testing the accuracy of the numerical calculations. Atomic units (a.u.) are assumed in all the results unless otherwise specified, and the particle has mass $m=1$.

The initial $(t=0)$ density is the form:

$$
\begin{aligned}
\rho(t=0)= & \left(\frac{1}{\sqrt{2 \pi}}\right)^{n} \prod_{i=1}^{n} \frac{1}{\sqrt{\chi_{i}}} \exp \left\{-\sum_{j, k=1}^{n} \frac{1}{2}\left[q_{j}-\overline{q_{j}}(0)\right]\right. \\
& \left.\times\left[q_{k}-\overline{q_{k}}(0)\right] \chi_{j k}^{-1}(0)\right\},
\end{aligned}
$$

where $n=4 ; \overline{q_{j}}(0)$ is the mean position of the Gaussian along the $j$ th coordinate at the initial time; $\chi_{j k}^{-1}(t)$ is the matrix inverse of the coordinate covariance matrix $\chi_{i j}$ where

$$
\chi_{i j}(t)=\left\langle\left(q_{i}-\overline{q_{i}(t)}\right)\left(q_{j}-\overline{q_{j}(t)}\right)\right\rangle .
$$

The principal values of the coordinate covariance matrix $\chi$ are denoted as $\chi_{i}$, which are obtained by diagonalizing the $\chi$ matrix. The principal axes of $\chi$ are initially $(t=0)$ at an angle of $\phi=30^{\circ}$ to the $q_{2}, q_{3}$ coordinate directions. We have chosen $\chi_{1}=1, \chi_{2}=1, \chi_{3}=0.7$, and $\chi_{4}=0.7$ at time, $t$ $=0$. Furthermore, we have $\overline{q_{1}}(0)=\overline{q_{2}}(0)=2.0, \overline{q_{3}}(0)=0.0$ and $\overline{q_{4}}(0)=-1.0$ and the initial group velocity is assumed to be zero along all of the coordinates. These initial conditions lead to the components of the $\chi$ matrix as

$$
\begin{aligned}
& \chi_{1 j}=\left\{\begin{aligned}
\chi_{1} & \text { if } j=1 \\
0 & \text { if } j \neq 1,
\end{aligned}\right. \\
& \chi_{4 j}=\left\{\begin{aligned}
\chi_{4} & \text { if } j=4 \\
0 & \text { if } j \neq 4,
\end{aligned}\right.
\end{aligned}
$$

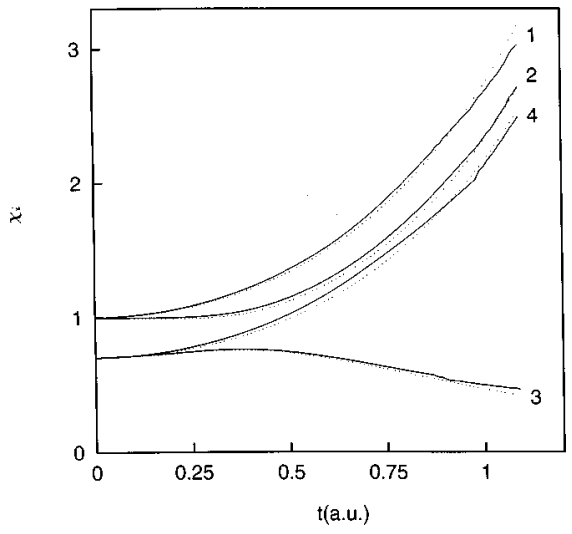

FIG. 2. $\chi_{i}$ where $i=1$ (curve 1), 2(curve 2), 3(curve 3), and 4(curve 4) for a $4 \mathrm{D}$ wave packet which jointly describes the spreading of the packet. The positive curvature of the potential along the $q_{3}$ direction leads to the contraction of the packet along $q_{3}$ ( $\chi_{3}$ decreases) whereas in the $q_{1}, q_{2}$, and $q_{4}$ directions the packet expands. Similar behavior of the calculated and analytical (dotted) results is found.

$$
\begin{aligned}
& \chi_{2 j}= \begin{cases}\chi_{2} \cos ^{2} \phi+\chi_{3} \sin ^{2} \phi & \text { if } j=2 \\
0 \quad \text { if } j \neq 2 \neq 3 & \\
\left(\chi_{2}-\chi_{3}\right) \cos \phi \sin \phi & \text { if } j=3,\end{cases} \\
& \chi_{3 j}= \begin{cases}\chi_{2} \sin ^{2} \phi+\chi_{3} \cos ^{2} \phi & \text { if } j=3 \\
0 & \text { if } j \neq 2 \neq 3 \\
\left(\chi_{2}-\chi_{3}\right) \cos \phi \sin \phi & \text { if } j=2 .\end{cases}
\end{aligned}
$$

The quadratic potential is of the general form:

$$
V=V_{0}+\sum_{i=1}^{n} \frac{1}{2} K_{i} q_{i}^{2}
$$

where $K_{i}$ is both positive and negative and $V_{0}=16$. We have chosen $K_{1}=-1, K_{2}=-1, K_{3}=1, K_{4}=-1$. Given the various parameters, particularly the negative curvatures of $V$, we expect rapid dispersion of the packet which is a good test of the algorithm.

Figures 1-3 display different characteristic parameters viz. $\left\langle q_{1}\right\rangle,\left\langle q_{2}\right\rangle,\left\langle q_{3}\right\rangle,\left\langle q_{4}\right\rangle, \chi_{1}, \chi_{2}, \chi_{3}, \chi_{4}$, and $\phi$. The angle $\phi$ is calculated as $\phi=\tan ^{-1}\left[-\left(\chi_{11}-\chi_{1}\right) / \chi_{12}\right]$. Comparison is made with the analytical results. The analytical solution for $\left\langle q_{j}(t)\right\rangle$ takes the form ${ }^{17}$

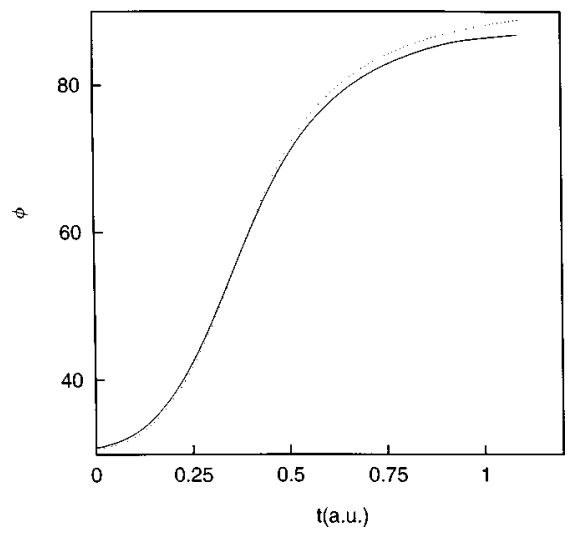

FIG. 3. Calculated rotation of the 4D wave packet $(\phi)$ along with its analytical value(dotted) in the $q_{2}-q_{3}$ plane. 
$\left\langle q_{j}(t)\right\rangle$

$$
= \begin{cases}q_{j}(0) \cosh \left(\omega_{j} t\right)+\overline{q_{j}}(0) \sinh \left(\omega_{j} t\right) / \omega_{j} & \text { if } K_{j}<0 \\ q_{j}(0) \cos \left(\omega_{j} t\right)+\overline{q_{j}}(0) \sin \left(\omega_{j} t\right) / \omega_{j} & \text { if } K_{j}>0,\end{cases}
$$

where

$$
\omega_{j}= \begin{cases}\sqrt{-K_{j}} & \text { if } K_{j}<0 \\ \sqrt{K_{j}} & \text { if } K_{j}>0 .\end{cases}
$$

Therefore, the expression for the potential [Eq. (56)] is such that only trigonometric functions appear in the equation for $\left\langle q_{j}(t)\right\rangle$ along the third coordinate axis $\left(q_{3}\right)$. However, the center of mass of the packet remains stationary along the $q_{3}$ axis (cf. Fig. 1 curve 3 ) because $q_{3}(0)=0$. Whereas along the $q_{1}$ and $q_{2}$ coordinate axes the center of mass propagates at a faster rate, because of the presence of the hyperbolic functions in their expressions (cf. Fig. 1, curves 1,2). The center of mass of the packet however, moves toward the negative $q_{4}$ axis [cf. Fig. 1 curve 4].

The analytical expression for $\chi_{i j}(t)$ takes, the form ${ }^{17}$

$$
\begin{aligned}
\chi_{i j}(t)= & \chi_{i j}(0) \cosh \left(\omega_{i} t\right) \cosh \left(\omega_{j} t\right) \\
& +\frac{\chi_{i j}^{-1}(0)}{4 \omega_{i} \omega_{j}} \sinh \left(\omega_{i} t\right) \sinh \left(\omega_{j} t\right),
\end{aligned}
$$

where $\chi_{i j}^{-1}$ is the matrix inverse of $\chi_{i j}$. In the above expression the hyperbolic trigonometric function is replaced by the corresponding trigonometric functions for $K_{j}>0$.

It is seen that the packet spreads rapidly in all the three directions viz. $q_{1}, q_{2}$, and $q_{4}$ directions (cf. Fig. 2 curves 1 , 2 , and 4) because of the antiharmonic character of the potential producing hyperbolic functions in the expressions for $\chi_{1}, \chi_{2}$, and $\chi_{4}$. At the same time, it shrinks along the $q_{3}$ direction[Fig. 2(3)] because of the trigonometric function in the expression for $\chi_{3}$. This process of spreading along the three directions causes the packet to reach the edges of the computation grid very fast. Also, the shrinkage along the other direction, $q_{3}$ is difficult to handle because $\chi / \Delta q_{3}$ becomes small in the course of time, where $\Delta q_{3}$ is the fixed grid size along the $q_{3}$ axis. In the calculation $\Delta q_{1}=0.389$, $\Delta q_{2}=0.389, \Delta q_{3}=0.271, \Delta q_{4}=0.333$ along with $\Delta t$ $=0.005$ and 220 time steps were carried out. The region of the discretized space was $-1.0 \leqslant q_{1} \leqslant 6.0,-1.0 \leqslant q_{2} \leqslant 6.0$, $-3.0 \leqslant q_{3} \leqslant 3.5$, and $-4.0 \leqslant q_{4} \leqslant 2.0$ leading, respectively, to $19,19,25$, and 19 discrete points in the $q_{1}, q_{2}, q_{3}$, and $q_{4}$ directions. The corresponding CPU time was 4 min on an IRIS Silicon Graphics Machine (Release 6.2).

The example we undertook thus poses a rather difficult numerical problem. However, the TD characteristic properties calculated following the present scheme clearly describe the spreading of the Gaussian packet along all the different directions over a time when substantial motion has occurred. The agreement between the analytical solution and the calculated values in Figs. 1-3 are excellent.

\section{B. Photodissociation}

The purpose of this subsection is not an exhaustive study, but rather an illustration of the proposed QFD method in the physically realistic and numerically challenging cases of photodissociation of $\mathrm{NOCl}$ and $\mathrm{NO}_{2} \cdot{ }^{13,14,35-42}$ These same systems have also been studied in the QFD formalism in the Lagrangian representation. ${ }^{18}$ The present systems are defined in terms of two radial coordinates and a single angular coordinate. In the case of $\mathrm{NOCl}$ the angular coordinate is Jacobi's angle whereas in the second case, it is the bending angle. However, here we reduce the three coordinates into two by freezing the angular variables that appear in the Hamiltonian. The angular variable is taken as that at their respective equilibrium values. A similar study in two-dimensional variables has been conducted for $\mathrm{CO}_{2}$ in the collinear arrangement which corresponds to taking the value of bending angle as frozen to zero. ${ }^{29}$ In spite of the approximation, the numerical problems studied here contain significant features of the physical behavior of the phenomena. The freezing of the angular variable eliminated some fine structure in the dynamics. This is reflected in the absorption spectrum for $\mathrm{NO}_{2}$. The present calculations serve as an illustration of the QFD methodology, and results from further three and higher coordinate systems will be reported later.

In the following subsections we first present a detailed model of the dissociation for $\mathrm{NOCl}$ and $\mathrm{NO}_{2}$ separately. The wave packet starts on the ground electronic potential surface, $S_{0}$ with the dynamical evolution followed on the excited electronic surface viz. $S_{1}$. The excitation from the $S_{0}$ to $S_{1}$ surface is assumed to occur instantaneously by a laser field so that initially the wave packet on the $S_{1}$ surface has the same shape as that on the $S_{0}$ surface.

\section{Dissociation of $\mathrm{NOCl}$}

The NOCl system is described by the Jacobi coordinates $\left(r_{d}, r_{v}, \theta\right)$ where the dissociation coordinate $r_{d}$ is the distance between the $\mathrm{Cl}$ and the center of mass of $\mathrm{N}-\mathrm{O}$, the vibrational coordinate $r_{v}$ is the $\mathrm{N}-\mathrm{O}$ distance, and $\theta$ the angle between $r_{d}$ and $r_{v}$. In this coordinate system the Hamiltonian of $\mathrm{NOCl}$ acting on the wave function is given as

$$
\begin{aligned}
H \psi= & -\frac{1}{2} \hbar^{2}\left[\left(\mu_{d} r_{d}\right)^{-1}\left(r_{d} \psi\right)_{r_{d} r_{d}}+\left(\mu_{v} r_{v}\right)^{-1}\left(r_{v} \psi\right)_{r_{v} r_{v}}\right. \\
& \left.+\left(I_{\theta} \sin \theta\right)^{-1} \frac{\partial}{\partial \theta} \sin (\theta) \frac{\partial}{\partial \theta} \psi\right]+V\left(r_{d}, r_{v}, \theta\right) \psi
\end{aligned}
$$

where

$$
\begin{aligned}
& \mu_{d}^{-1}=\left(m_{\mathrm{N}}+m_{\mathrm{O}}\right)^{-1}+m_{\mathrm{cl}}^{-1}, \\
& \mu_{v}^{-1}=m_{\mathrm{N}}^{-1}+m_{\mathrm{O}}^{-1}, \\
& I_{\theta}^{-1}=\left(\mu_{v} r_{v}^{2}\right)^{-1}+\left(\mu_{d} r_{d}^{2}\right)^{-1}
\end{aligned}
$$

and the subscripts $r_{d}$ or $r_{v}$ refer to the corresponding differentiation.

The $\theta$ angle is frozen at the equilibrium value $\theta_{\mathrm{eq}}$ $=127.4^{\circ} .{ }^{35}$ Thus, we have a two-dimensional equation as

$$
\begin{aligned}
i \hbar \frac{\partial \psi}{\partial t}=H \psi= & -\frac{\hbar^{2}}{2}\left[\left(\mu_{d} r_{d}\right)^{-1}\left(r_{d} \psi\right)_{r_{d} r_{d}}\right. \\
& \left.+\left(\mu_{v} r_{v}\right)^{-1}\left(r_{v} \psi\right)_{r_{v} r_{v}}\right]+V\left(r_{d}, r_{v}, \theta_{\mathrm{eq}}\right) \psi .
\end{aligned}
$$

With the substitution $\Psi=r_{d} r_{v} \psi$, Eq. (58) reduces to ${ }^{18}$ 


$$
i \hbar \frac{\partial \Psi}{\partial t}=-\frac{\hbar^{2}}{2}\left[\frac{1}{\mu_{d}} \Psi_{r_{d^{r}}}+\frac{1}{\mu_{v}} \Psi_{r_{v} r_{v}}\right]+V \Psi
$$

and multiplication of both sides by $r_{0}^{2} \mu_{v}$, leads to the equation in nondimensional variables

$$
i \frac{\partial \Psi}{\partial t^{\prime}}=-\frac{1}{2}\left[\frac{\partial^{2} \Psi}{\partial x^{2}}+\frac{\partial^{2} \Psi}{\partial y^{2}}\right]+V_{1} \Psi
$$

where

$x=\frac{r_{d} \sqrt{\mu_{d}}}{r_{\mathrm{eq}} \sqrt{\mu_{v}}}, \quad y=\frac{r_{v}}{r_{\mathrm{eq}}}, \quad t^{\prime}=\frac{\hbar t}{r_{\mathrm{eq}}^{2} \mu_{v}}, \quad V_{1}=V r_{\mathrm{eq}}^{2} \mu_{v} / \hbar^{2}$.

This equation has the appearance of a single effective particle moving in two dimensions. In the above equations $r_{\text {eq }}$ is a length scale and is taken to be the equilibrium value of $r_{v}$ on the $S_{0}$ surface. The $S_{0}$ potential surface in terms of $r_{1}$, $r_{2}$, and $r_{3}$ given in Ref. 35 is

$$
\begin{aligned}
V\left(r_{1}, r_{2}, r_{3}\right)= & \frac{1}{2} K_{1}\left(r_{1}-r_{1}^{\mathrm{eq}}\right)^{2}+\frac{1}{2} K_{2}\left(r_{2}-r_{2}^{\mathrm{eq}}\right)^{2} \\
& +\frac{1}{2} K_{3}\left(r_{3}-r_{3}^{\mathrm{eq}}\right)^{2}+K_{23}\left(r_{2}-r_{2}^{\mathrm{eq}}\right)\left(r_{3}-r_{3}^{\mathrm{eq}}\right),
\end{aligned}
$$

where $r_{1}$ is the $\mathrm{N}-\mathrm{O}$ bond, $r_{2}$ the $\mathrm{Cl}-\mathrm{N}$ bond, and $r_{3}$ the $\mathrm{Cl}-\mathrm{O}$ bond. Because this potential has a deep minimum, the initial ground state wave function is constructed from the quadratic approximation of the form given in Eq. (63). The Appendix shows the derivation of the quadratic approximation as

$$
\begin{aligned}
V_{1}(x, y)= & \frac{1}{2} K_{11}\left(x-x^{\mathrm{eq}}\right)^{2}+\frac{1}{2} K_{22}\left(y-y^{\mathrm{eq}}\right)^{2} \\
& +K_{12}\left(x-x^{\mathrm{eq}}\right)\left(y-y^{\mathrm{eq}}\right)
\end{aligned}
$$

where $K_{11}, K_{22}, K_{12}, x^{\mathrm{eq}}$, and $y^{\mathrm{eq}}$ are given in the Appendix.

Having simplified the $S_{0}$ surface in Eq. (64), the ground state is

$$
\begin{aligned}
\Psi\left(S_{0}\right)= & \frac{1}{(2 \pi)^{1 / 2}} \frac{1}{\chi_{1}^{1 / 4}} \frac{1}{\chi_{2}^{1 / 4}} \exp \left(-\frac{1}{4}\left[\left(x-x^{\mathrm{eq}}\right)^{2} \chi_{11}^{-1}\right.\right. \\
& \left.\left.+\left(y-y^{\mathrm{eq}}\right)^{2} \chi_{22}^{-1}+2\left(x-x^{\mathrm{eq}}\right)\left(y-y^{\mathrm{eq}}\right) \chi_{12}^{-1}\right]\right)
\end{aligned}
$$

where $\chi_{1}$ and $\chi_{2}$ in the above expression are related to the eigenvalues $\omega_{1}$ and $\omega_{2}$ of the $2 \times 2 K$ matrix (elements are $K_{11}, K_{22}$, and $\left.K_{12}=K_{21}\right)$ as

$$
\chi_{1}=\frac{1}{2 \sqrt{\omega_{1}}}, \quad \chi_{2}=\frac{1}{2 \sqrt{\omega_{2}}} .
$$

$\chi^{-1}$ is the inverse of the matrix $\chi$ whose elements are given by

$$
\begin{aligned}
& \chi_{11}=\chi_{1} \cos ^{2} \beta+\chi_{2} \sin ^{2} \beta, \\
& \chi_{22}=\chi_{1} \sin ^{2} \beta+\chi_{2} \cos ^{2} \beta, \\
& \chi_{12}=\left(\chi_{1}-\chi_{2}\right) \cos \beta \sin \beta,
\end{aligned}
$$

TABLE I. Parameters for the $\mathrm{NOCl}$ and $\mathrm{NO}_{2}$ systems in atomic units.

\begin{tabular}{cccc}
\hline \hline Parameters $(\mathrm{NOCl})$ & Values & Parameters $\left(\mathrm{NO}_{2}\right)$ & Values \\
\hline$r_{1}^{\text {eq }}$ & 2.155 & $r_{1}^{\text {eq }}$ & 4.29 \\
$r_{2}^{\text {eq }}$ & 3.729 & $r_{2}^{\text {eq }}$ & 4.29 \\
$r_{3}^{\text {eq }}$ & 4.989 & $r_{\text {eq }}$ & 4.29 \\
$r_{0}$ & 2.155 & $r_{0}$ & 4.29 \\
$K_{1}$ & 0.8987 & $C_{20}$ & 0.1261 \\
$K_{2}$ & 0.0874 & $C_{11}$ & 0.0659 \\
$K_{3}$ & 0.1137 & $\ldots$ & $\ldots$ \\
$K_{23}$ & 0.0122 & $\ldots$ & $\ldots$ \\
$r_{v}^{\text {eq }}$ & 2.155 & $\ldots$ & $\ldots$ \\
$r_{d}^{\text {eq }}$ & 4.314 & $\ldots$ & $\ldots$ \\
$\theta_{\text {eq }}$ & $127.4^{\circ}$ & $\theta_{\text {eq }}$ & $133.63^{\circ}$ \\
$A$ & 0.9696 & $\ldots$ & $\ldots$ \\
$B$ & -0.2103 & $\ldots$ & $\ldots$ \\
$C$ & 0.9871 & $\ldots$ & $\ldots$ \\
$D$ & 0.3393 & $\ldots$ & $\ldots$ \\
\hline \hline
\end{tabular}

where $\beta$ is the orientation of the first principal axis of the initial wave packet with respect to the $q_{1}$ coordinate line. It is defined as

$$
\beta=\tan ^{-1}\left(\frac{K_{11}-\omega_{1}}{K_{12}}\right) .
$$

The parameters that appear in the above equations for $\mathrm{NOCl}$ are listed in Tables I and II.

The $S_{1}$ surface for NOCl is taken as ${ }^{18,35}$

$$
V=\sum_{i=1}^{4} a_{i} q_{v}^{i}+\left(1-q_{d}\right) \sum_{i=0}^{3} \sum_{j=0}^{4} \sum_{k=0}^{6} c_{i j k} q_{v}^{i} q_{d}^{j} q_{\theta}^{k},
$$

where

$$
\begin{aligned}
& q_{d}=1-\exp \left[-\alpha\left(r_{d}-r_{d_{p}}^{\mathrm{eq}}\right)\right]=1-\exp \left[-\alpha^{\prime}\left(x-x_{p}^{\mathrm{eq}}\right)\right], \\
& q_{v}=r_{v}-r_{v_{p}}^{\mathrm{eq}}=a\left(y-y_{p}^{\mathrm{eq}}\right), \\
& q_{\theta}=e^{-\beta \cos \theta}-e^{-\beta \cos \theta_{e_{p}}}
\end{aligned}
$$

where

$$
\alpha^{\prime}=\alpha r_{0} \frac{\sqrt{\mu_{v}}}{\sqrt{\mu_{d}}}, \quad x_{p}^{\mathrm{eq}}=\frac{r_{d}^{\mathrm{eq}} \sqrt{\mu_{d}}}{r_{0} \sqrt{\mu_{v}}}, \quad a=r_{0}, \quad y_{p}^{\mathrm{eq}}=r_{v_{p}}^{\mathrm{eq}} a .
$$

TABLE II. Parameters for the $\mathrm{NOCl}$ and $\mathrm{NO}_{2}$ systems in dimensionless units.

\begin{tabular}{cccc}
\hline \hline $\begin{array}{c}\text { Parameters } \\
(\mathrm{NOCl})\end{array}$ & Values & Parameters $\left(\mathrm{NO}_{2}\right)$ & Values \\
\hline$K_{11}$ & 29402.833 & $K_{11}$ & 1171606.24 \\
$K_{22}$ & 2022.1775 & $K_{22}$ & 1171606.24 \\
$K_{12}$ & 2022.1775 & $K_{12}$ & 306141.36 \\
$x^{\mathrm{eq}}$ & 2.953 & $x^{\mathrm{eq}}$ & 1.0 \\
$y^{\mathrm{eq}}$ & 1.0 & $y^{\text {eq }}$ & 1.0 \\
$\chi_{1}$ & $9.62 E-04$ & $\chi_{1}$ & $4.11 E-04$ \\
$\chi_{2}$ & $2.92 E-03$ & $\chi_{2}$ & $5.37 E-04$ \\
$\omega_{1}$ & 270350.91 & $\omega_{1}$ & 1477747.59 \\
$\omega_{2}$ & 29385.86 & $\omega_{2}$ & 865464.88 \\
$\beta$ & $89.519^{\circ}$ & $\beta$ & $45^{\circ}$ \\
\hline \hline
\end{tabular}


In the above potential $\theta_{e_{p}}=127.4, r_{v_{p}}^{\mathrm{eq}}=2.136$, and $r_{d_{p}}^{\mathrm{eq}}$ $=4.315$. The other parameters are given in Ref. 35 .

\section{Dissociation of $\mathrm{NO}_{2}$}

Here we use $r_{1}$ and $r_{2}$ as the coordinates for the two $\mathrm{N}-\mathrm{O}$ bond lengths. The action of full Hamiltonian on the wave function is 36

$$
\begin{aligned}
H \psi= & -\frac{1}{2} \hbar^{2}\left[\mu_{-1}\left(\psi_{r_{1} r_{1}}+\psi_{r_{2} r_{2}}\right)+\left(I_{\theta} \sin \theta\right)^{-1}\right. \\
& \times\left(\sin \theta \psi_{\theta}\right)_{\theta}+2 m_{N}^{-1} \cos \theta \psi_{r_{1} r_{1}}+\left(m_{\mathrm{N}} r_{1} r_{2}\right)^{-1} \\
& \times\left(\cos \theta \sin ^{-1} \theta\left(\sin \theta \psi_{\theta}\right)_{\theta}\right. \\
& \left.+\sin ^{-1} \theta\left(\sin \theta(\cos \theta \psi)_{\theta}\right)_{\theta}\right)-m_{N}^{-1}\left(r_{2}^{-1}(\sin \theta \psi)_{\theta_{r_{1}}}\right. \\
& \left.\left.+r_{1}^{-1}(\sin \theta \psi)_{\theta_{r_{2}}}\right)\right]+V\left(r_{1}, r_{2}, \theta\right) \psi .
\end{aligned}
$$

where the subscripts $r_{1}, r_{2}$, and $\theta$ indicate partial derivatives and

$$
\mu^{-1}=m_{\mathrm{N}}^{-1}+m_{\mathrm{O}}^{-1}, \quad I_{\theta}^{-1}=\mu^{-1}\left(r_{1}^{-2}+r_{2}^{-2}\right) .
$$

The angle $\theta$ is fixed at $\theta_{\text {eq }}$, yielding the two-dimensional (2D) equation as

$$
\begin{aligned}
i \hbar \frac{\partial \psi}{\partial t}=H \psi= & -\frac{1}{2} \hbar^{2}\left[\mu^{-1} \psi_{r_{1} r_{1}}+\mu_{-1} \psi_{r_{2} r_{2}}\right] \\
& +V\left(r_{1}, r_{2}, \theta_{\mathrm{eq}}\right) \psi .
\end{aligned}
$$

To obtain a simpler form, we multiply Eq. (68) by $r_{\text {eq }}^{2} \mu$ leading to

$$
i \frac{\partial \psi}{\partial t^{\prime}}=-\frac{1}{2}\left[\frac{\partial^{2} \psi}{\partial x^{2}}+\frac{\partial^{2} \psi}{\partial y^{2}}\right]+V_{1} \psi
$$

With the definitions

$$
t^{\prime}=\frac{\hbar t}{r_{\mathrm{eq}}^{2}}, \quad x=\frac{r_{1}}{r_{\mathrm{eq}}}, \quad y=\frac{r_{2}}{r_{\mathrm{eq}}}, \quad V_{1}=V r_{\mathrm{eq}}^{2} \mu / \hbar^{2} .
$$

Here $r_{\mathrm{eq}}$ is taken to be $r^{\mathrm{eq}}=r_{1}^{\mathrm{eq}}=r_{2}^{\mathrm{eq}}$, which is the equilibrium bond length for $\mathrm{NO}_{2}$ on the $S_{0}$ surface. The $S_{0}$ potential energy is given as the quadratic function: ${ }^{36}$

$$
\begin{aligned}
V\left(r_{1}, r_{2}\right)= & C_{20}\left[\left(r_{1}-r^{\mathrm{eq}}\right)^{2}+\left(r_{2}-r^{\mathrm{eq}}\right)^{2}\right] \\
& +C_{11}\left(r_{1}-r^{\mathrm{eq}}\right)\left(r_{2}-r^{\mathrm{eq}}\right) .
\end{aligned}
$$

The substitution of $r_{1}$ and $r_{2}$ from Eq. (70) into Eq. (71) yields

$$
\begin{aligned}
V_{1}(x, y)= & \frac{1}{2} K_{11}\left(x-x^{\mathrm{eq}}\right)^{2}+\frac{1}{2} K_{22}\left(y-y^{\mathrm{eq}}\right)^{2} \\
& +K_{12}\left(x-x^{\mathrm{eq}}\right)\left(y-y^{\mathrm{eq}}\right),
\end{aligned}
$$

where $K_{11}=K_{22}=2 C_{20} r_{0}^{4} \mu$ and $K_{12}=C_{11} r_{0}^{4} \mu$.

The ground state for the $S_{0}$ surface is given again by the same Gaussian form as in Eq. (65). The parameters that appear for the $\mathrm{NO}_{2}$ molecule are listed in Tables I and II.

The $S_{1}$ surface for $\mathrm{NO}_{2}$ is given as ${ }^{36}$

$$
\begin{aligned}
V= & V_{\mathrm{NO}}\left(r_{1}\right)+V_{\mathrm{NO}}\left(r_{2}\right)+\left[1-Q_{a}\left(r_{1}\right)\right]\left[1-Q_{a}\left(r_{2}\right)\right] \\
& \times \sum_{k, l, m} b_{k l m} Q_{a}\left(r_{1}\right)^{k} Q_{a}\left(r_{2}\right)^{l} Q_{b}(\theta)^{m},
\end{aligned}
$$

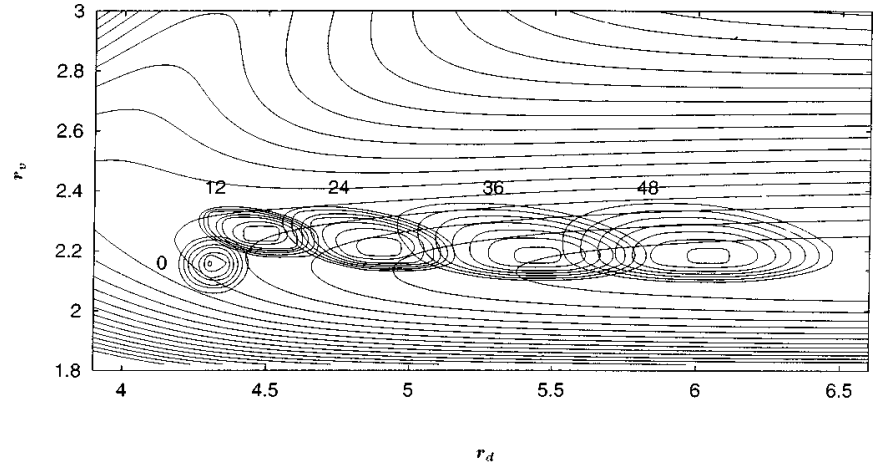

FIG. 4. Contour plot of the $S_{1}$ potential-energy surface as a function of $r_{d}$ and $r_{v}$ for fixed $\theta=127.4^{\circ}$. Superimposed are contours of the wave packet for the $\mathrm{NOCl}$ molecule at five different times (in fs). As $\log \rho$ is actually the variable in the dynamics, very few points are needed to represent it due to the slow variation (cf. also Fig. 10). This results in minor graphical artifacts in the contour lines.

where

$$
\begin{aligned}
& Q_{a}(r)=1-e^{-\beta\left(r-r_{e_{p}}\right)}=1-e^{-\beta^{\prime}\left(x-x_{e_{p}}\right),} \\
& Q_{b}(\theta)=\alpha_{1}\left(\theta-\theta_{e}\right)+\alpha_{2}\left(\theta-\theta_{e}\right)^{2}+\alpha_{3}\left(\theta-\theta_{e}\right)^{3}, \\
& V_{\mathrm{NO}}(r)=a_{0}+a_{2} Q_{a}(r)^{2}+a_{3} Q_{a}(r)^{3}+a_{4} Q_{a}(r)^{4},
\end{aligned}
$$

and $\beta^{\prime}=\beta r^{\mathrm{eq}}, \quad x_{e_{p}}=r_{e_{p}} / r^{\mathrm{eq}}, \quad \theta_{e}=101.889^{\circ}, \quad r_{e_{p}}=2.4125$. The other parameters are given in Ref. 36.

\section{Results for dissociation}

\section{1. $\mathrm{NOCl}$}

Figure 4 displays five contours of density $\rho\left(r_{d}, r_{v}\right)$ at five different times viz. $0,12,24,36$ and 48 fs. The contours of the $S_{1}$ potential energy surface as a function of $r_{d}$ and $r_{v}$ and for fixed $\theta=127.4^{\circ}$ are also shown in Fig. 4. It is readily seen that the initially $(t=0)$ compact wave packet centered at $r_{d}=4.31$, has propagated rapidly along the dissociative coordinate $r_{d}$, whereas along the vibrational coordinate $r_{v}$ there is relatively little change except an initial drift along at 12 fs. During the entire process the compact form of the wave packet is retained, but it has spread in both the $r_{v}$ and $r_{d}$ directions. The same behavior of the density contours is observed in Fig. 1 of Ref. 35 and 18. The calculations used a grid spanning the region $2.2 \leqslant x \leqslant 5.5,0.8 \leqslant y \leqslant 1.7$ and with 81 points in each direction. This leads to $\Delta x=0.041$ and $\Delta y=0.01$. The $\Delta t=7.7 \times 10^{-4}$ fs is used and the calculations are carried on for about 60000 time steps. The corresponding CPU time was 15 min on an IRIS Silicon Graphics Machine (Release 6.2).

To see how the time evolved state correlates to its initial $(t=0)$ form we calculated the correlation function as

$$
C(t)=\int \psi^{*}(0) \psi(t) d x d y
$$



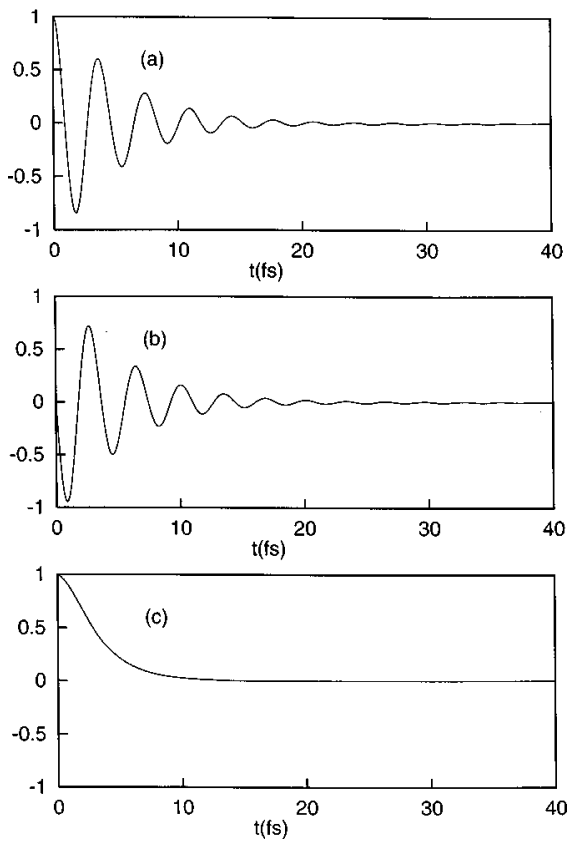

FIG. 5. Real (a) and imaginary (b) parts of the correlation function of $\mathrm{NOCl}$ with respect to time (in $\mathrm{fs}$ ). The survival probability (c) of the initial $(t$ $=0$ ) packet falls off monotonically.

where $\psi=\Psi / r_{v} r_{d}$ for NOCl [Eq. (59)]. This requires the phase function $S(x, y)$, which has been calculated as follows. The discrete form of Eq. (2) can be written as

$$
S_{i, j}^{n+1}=S_{i, j}^{n}-\frac{\left(v_{i, j}\right)^{2}}{2} \delta t-\left(V_{i, j}+\left(V_{q}\right)_{i, j}\right) \delta t,
$$

where $i$ and $j$ are the indices of the 2D discrete grid. Initially $(t=0, n=1), S_{i, j}^{1}=0$. Thus, we solve Eqs. (5) and (6) for $\rho$, $j_{x}$, and $j_{y}$, and then calculate $v_{x}=j_{x} / \rho ; v_{y}=j_{y} / \rho$ which are used to obtain the phase form the above equation. The survival probability of the initial $(t=0)$ state is given by

$$
P(t)=|C(t)|^{2} .
$$

Figure 5 shows the real (curve a) and the imaginary (curve b) part of $C(t)$ which are oscillatory forms while $P(t)$ (curve c) rapidly decreases monotonically to zero.

We also calculated the absorption spectrum as ${ }^{27}$

$$
\sigma(\omega)=\omega \operatorname{Re}\left(\int\langle\Psi(0) \mid \Psi(t)\rangle e^{i \omega t} \exp \left(-\frac{t^{2}}{\tau^{2}}\right) d t\right) .
$$

The quantity $\tau$ characterizes the finite resolution of the spectrum which is taken as $67 \mathrm{fs}^{36}$ for both molecular systems. The choice of the window function corresponds to a convolution of the spectrum with a Gaussian. Figure 6 shows the spectrum for NOCl. The broad spectrum corresponds to fast and direct dissociation. ${ }^{35}$ Some differences are evident upon close comparison of our spectrum and that in Ref. 35, due to freezing of the angle at a constant value in our calculation. However, the peak position $(1.2 \mathrm{eV})$ agrees well with the existing result.

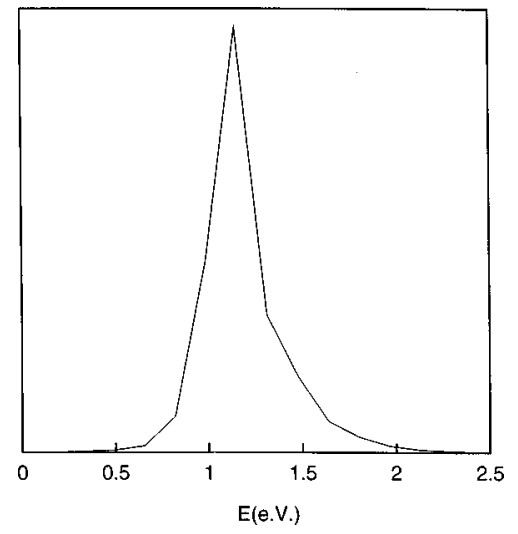

FIG. 6. The photoabsorption spectrum of NOCl. The peak position $(1.2 \mathrm{eV})$ is in good agreement with the existing result.

\section{2. $\mathrm{NO}_{2}$}

Figure 7 displays the five contour plots of density corresponding to $0(\mathrm{a}), 12(\mathrm{~b}), 24(\mathrm{c}), 36(\mathrm{~d})$, and 48(e) fs time in the $r_{1}, r_{2}$ coordinates. The contours for the $S_{1}$ potential surface of $\mathrm{NO}_{2}$ molecule in the $r_{1}, r_{2}$ coordinates and at fixed $\theta$ $=133.63^{\circ}$ have been projected onto each of the above plots. The wave packet centered at $r_{1}=r_{2}=4.29$ has split symmetrically after 12 fs and finally fragments into two equal halves. The packet moves in the direction of the symmetric stretch coordinate $r_{1}+r_{2}$ as noticed earlier. ${ }^{36}$ The symmetric nature of the wave packet over time clearly demonstrates that the QFD method has introduced no instability, as the symmetry was not imposed in the numerical calculations. The calculations used a grid spanning the region $0.905 \leqslant x \leqslant 2.3$, $0.905 \leqslant y \leqslant 2.3$ and with 131 points in each direction. This leads to $\Delta x=0.01$ and $\Delta y=0.01$. The $\Delta t=3.05 \times 10^{-3}$ fs is used and the calculations are carried on for about 16000 time steps. The corresponding CPU time was $20 \mathrm{~min}$ on an IRIS Silicon Graphics Machine (Release 6.2)

Figure 8 presents the real (curve a) and the imaginary (curve b) part of the correlation function along with the survival probability (curve $\mathrm{c}$ ) for the initial $(t=0)$ wave packet. The behavior is similar to that of NOCl. The absorption spectrum in Fig. 9 indicates the fast dissociation with the center peaked near $3.6 \mathrm{eV}$. Compared to the results of Ref. 36 we did not observe any fine structure in the spectrum, presumably because of the freezing of the molecule at a constant angle. Nevertheless, the peak value agrees well with that of Refs. 36 and 18. The relatively low resolution of $\mathrm{NO}_{2}$ spectrum compared to that of $\mathrm{NOCl}$ is because of the lesser number of sample datas for the former molecule.

\section{CONCLUSION}

In the present paper we have presented a new technique for solving the hydrodynamical form of Schrödinger's equation along with its application to the motion of a Gaussian wave packet in four-dimensions and to the photodissociation of $\mathrm{NOCl}$ and $\mathrm{NO}_{2}$ molecule. The new scheme is general and applicable to any number of dimensions in both the Eulerian and Lagrangian forms. The application of the formulation in the Lagrangian form is presented in a separate paper. ${ }^{18}$ In the Eulerian form one should use due care on the choice of the 

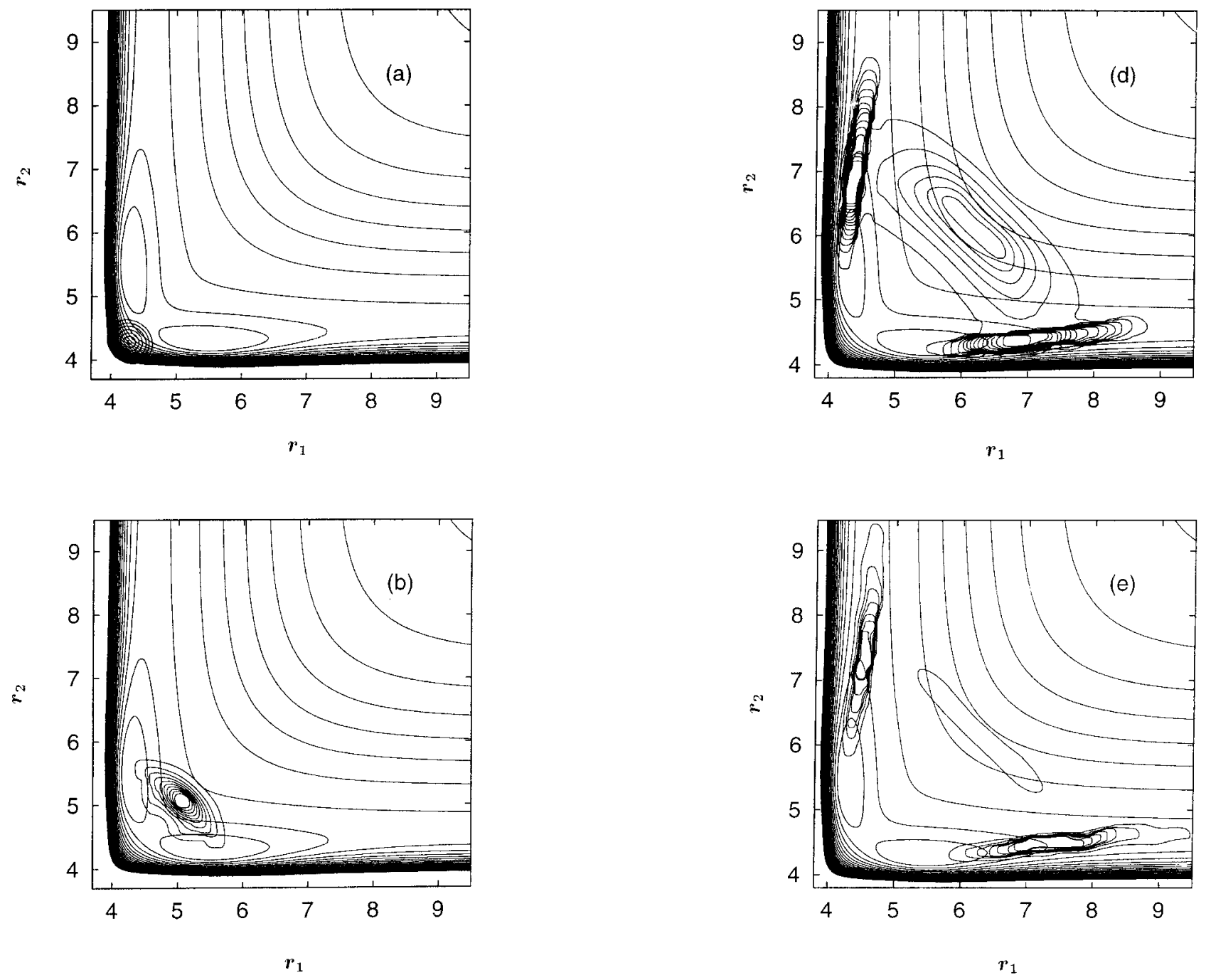

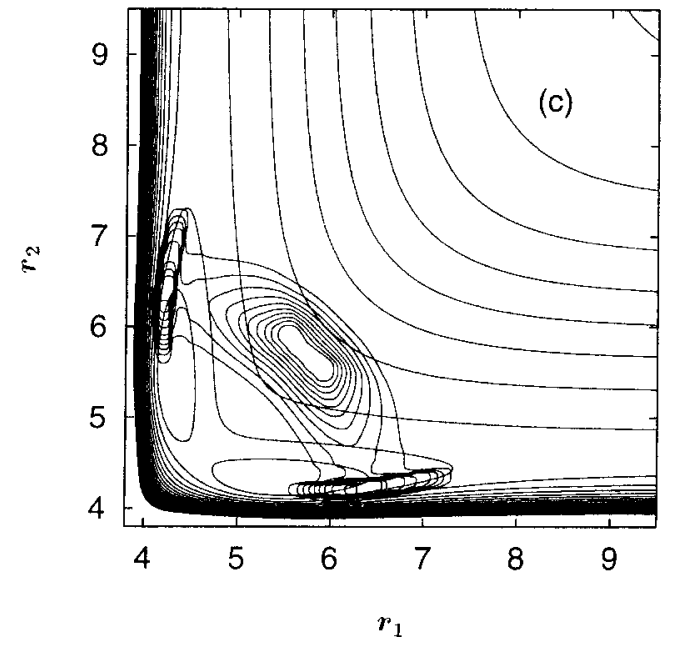

grid size which is kept fixed over time. Since the dynamics in the present technique directly involves a velocity defined at each grid point by $v=i / \rho$, the choice of the grid should be made such that the density is always substantial. At the same time, the domain size should be sufficiently large to avoid any spurious effect in the solution arising out of reflection by the edges of the grid. An appropriate complex term could be added to the potential in the boundary region to absorb the flux and avoid this problem. From another perspective, since a velocity term is associated with each grid point one can find a law governing the motion of these points thereby again avoiding boundary effects. The extremely small values of $\rho$ as a result of the Gaussian decay, fall below machine accuracy in the far regions of the domain. This creates problems 

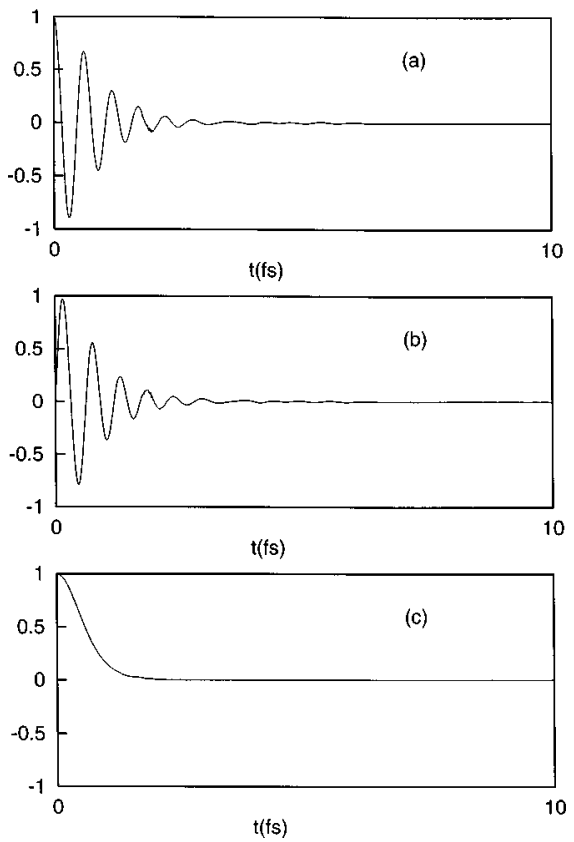

FIG. 8. Real (a) and imaginary (b) parts of the correlation function of $\mathrm{NO}_{2}$ with respect to time (in $\mathrm{fs}$ ). The survival probability (c) of the initial $(t$ $=0$ ) packet falls off monotonically.

in evaluating $V_{q}=-\frac{1}{2} \nabla^{2} \rho^{1 / 2} / \rho^{1 / 2}$. These problems are avoided by working with $\log \rho$ in the equivalent expression $V_{q}=-\frac{1}{2}\left[\nabla^{2} \log \rho^{1 / 2}+\left(\nabla \log \rho^{1 / 2}\right)^{2}\right]$. In addition, in the inner regions, $\log \rho$ is more readily represented numerically as it is a more slowly varying function than $\rho$. For example, at the initial time, $\rho$ is a Gaussian which leads to $\log \rho$ to be only a quadratic. The same simple polynomial trend at all times makes it possible to represent $\log \rho$ by a very coarse grid. This attractive computational feature also results in the minor graphical artifacts in the contour plots in Figs. 4 and 7. A similar coarse grid benefit is expected in rewriting the mass conservation equation as $\partial \log \rho / \partial t=-\nabla \cdot(\log \rho \mathbf{v})$ $+(1-\log \rho) \boldsymbol{\nabla} \cdot \mathbf{v}$ and working with $\log \rho$. In this new form, $\log \rho$ is still convected with the velocity $\mathbf{v}$ in the presence of the source term $(1-\log \rho) \boldsymbol{\nabla} \cdot \mathbf{v}$.

A high value of the quantum potential is responsible for the development of nodes at any point in the space and vice

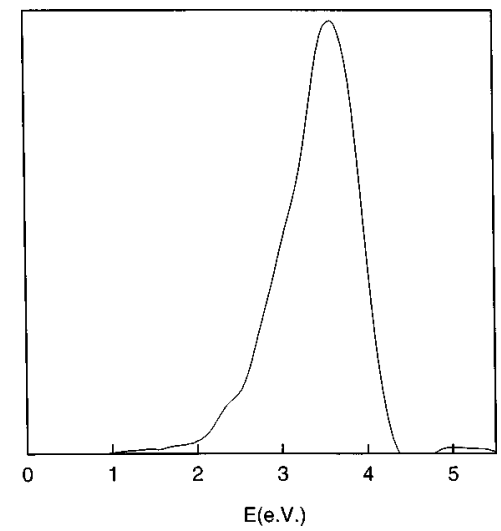

FIG. 9. The photoabsorption spectrum of $\mathrm{NO}_{2}$. The peak position $(3.6 \mathrm{eV})$ is in good agreement with the existing result.
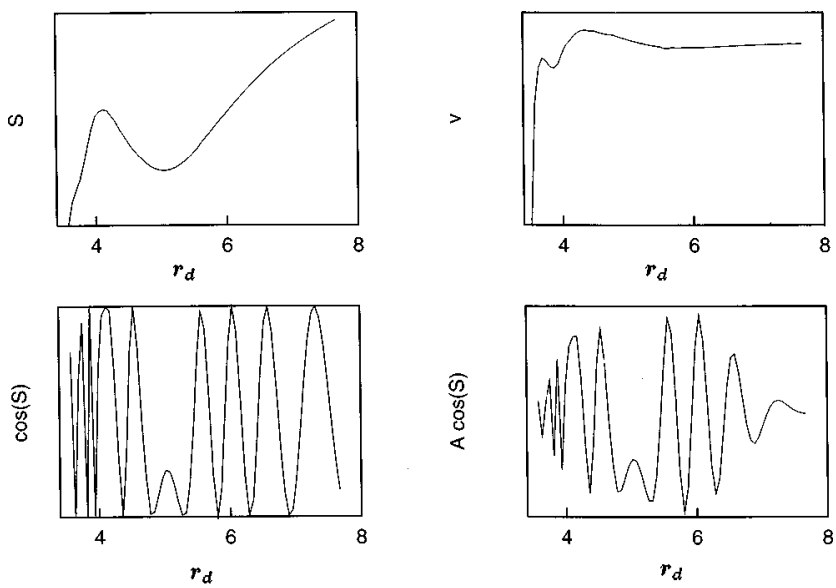

FIG. 10. The phase $(S)$, velocity $(v), \cos (S)$, and $A \cos (S)$ for NOCl plotted along $r_{d}$ when $r_{v}=2.427$ a.u. at $t=48 \mathrm{fs}$. Notice that both $S$ and $v$ are smooth whereas $\cos (S)$ and $A \cos (S)$ are oscillatory.

versa. This can be handled computationally by putting a lower limit (e.g., the lowest value permitted by the computer) on the value of $\rho$, which in our present calculation is taken as $1 \times 10^{-21}$. This does not alter the essential physical nature of the problems, as for example in the study of the dissociation of $\mathrm{NO}_{2}$ molecule where the development of a node separates the initial packet into two equal halves.

The successful application of QFD to the fourdimensional rapidly spreading dynamics and to the photodissociation of $\mathrm{NOCl}$ and $\mathrm{NO}_{2}$ indicates the potential capability of the QFD method. The dissociation calculation for NOCl and $\mathrm{NO}_{2}$ took 15 and $20 \mathrm{CPU}$ min, respectively, on an IRIS Silicon Graphics Machine(Release 6.2). Similarly, the wave packet dynamics calculation in the quadratic potential in four dimensions took $4 \mathrm{CPU}$ min on the same machine.

The QFD, the alternating direction within the Eulerian description, and the explicit time integrator collectively lead to the success and promise of the method. QFD permits one to work near monotonous amplitude and phase. The reduction of the number of discrete points as compared to what would be needed to represent the oscillatory real and imaginary parts of the complex wave function is illustrated in Fig. 10. Figure 10 contrasts the typical smooth and monotonic behavior of the phase $S$ and the velocity in the $r_{d}$ direction $v=\hbar\left(\partial S / m \partial r_{d}\right)$ with the typical oscillations in the functions $\cos (S)$ and $\operatorname{Re} \Psi \equiv A \cos (S)$. Figure 10 shows the cross sections along $r_{v}=2.427$ a.u. and at time $48 \mathrm{fs}$ for $\mathrm{NOCl}$ case. The oscillations in the $r_{v}$ direction are similar. Within the QFD scheme one needs to discretize the smooth $S$ or $v$ as opposed to the need of discretization of the oscillatory real and imaginary parts of $e^{i S}$ and/or $\Psi=A e^{i S}$. The advantages of QFD in terms of the sparseness of the discrete points is apparent and the number of the discrete points is expected to be at least an order of magnitude smaller than what would be needed to represent the oscillatory real and imaginary parts of the complex wave function.

The Eulerian description being based on a fixed grid allows one to work with a regular grid. This feature is key for the stability and implementation of the alternating direction method (ADM). The ADM in turn converts an $n$ coor- 
dinates problem into an assembly of one coordinate problems which is a most desirable capability in view of the multidimensional problems in quantum mechanics. Finally, the explicit time integrator used both pressures mass and flux along with making the computational effort grow only linearly with the number of discrete points.

\section{ACKNOWLEDGMENTS}

The authors (B.D.) thank the Koc University research fund, Istanbul, the National Research Council of Turkey, TUBA (A.A.), and the National Science Foundation (B.D., H.R.) for support of this work.

\section{APPENDIX}

We have approximated the $S_{0}$ potential surface of $\mathrm{NOCl}$ [Eq. (63)] into a quadratic form as described below by

The variables $r_{1}, r_{2}$, and $r_{3}$ are related to $r_{d}, r_{v}$, and $\theta$

$$
\begin{aligned}
& r_{1}=r_{v}, \\
& r_{2}^{2}=\left(\frac{m_{\mathrm{N}}}{m_{\mathrm{O}}+m_{\mathrm{N}}}\right)^{2} r_{v}^{2}+r_{d}^{2}+2 r_{v} r_{d} \cos \theta \frac{m_{\mathrm{O}}}{m_{\mathrm{O}}+m_{\mathrm{N}}}, \\
& r_{3}^{2}=\left(\frac{m_{\mathrm{N}}}{m_{\mathrm{O}}+m_{\mathrm{N}}}\right)^{2} r_{v}^{2}+r_{d}^{2}-2 r_{d} r_{v} \cos \theta \frac{m_{\mathrm{N}}}{m_{\mathrm{O}}+m_{\mathrm{N}}} .
\end{aligned}
$$

Since the $S_{0}$ wave packet is the harmonic ground vibrational state, we will Taylor expand to obtain a harmonic $S_{0}$ potential in the new coordinates,

$$
\begin{gathered}
r_{2}=r_{2}^{\mathrm{eq}}+\left.\frac{\partial r_{2}}{\partial r_{d}}\right|_{\mathrm{eq}}\left(r_{d}-r_{d}^{\mathrm{eq}}\right)+\left.\frac{\partial r_{2}}{\partial r_{v}}\right|_{\mathrm{eq}}\left(r_{v}-r_{v}^{\mathrm{eq}}\right), \\
r_{3}=r_{3}^{\mathrm{eq}}+\left.\frac{\partial r_{3}}{\partial r_{d}}\right|_{\mathrm{eq}}\left(r_{d}-r_{d}^{\mathrm{eq}}\right)+\left.\frac{\partial r_{3}}{\partial r_{v}}\right|_{\mathrm{eq}}\left(r_{v}-r_{v}^{\mathrm{eq}}\right),
\end{gathered}
$$

which can be rewritten as

$$
\begin{aligned}
& r_{2}-r_{2}^{\mathrm{eq}}=A\left(r_{d}-r_{d}^{\mathrm{eq}}\right)+B\left(r_{v}-r_{v}^{\mathrm{eq}}\right) \\
& r_{3}-r_{3}^{\mathrm{eq}}=C\left(r_{d}-r_{d}^{\mathrm{eq}}\right)+D\left(r_{v}-r_{v}^{\mathrm{eq}}\right) .
\end{aligned}
$$

The expressions for $A, B, C$ and $D$ are given by

$$
\begin{aligned}
& A=\left.\frac{\partial r_{2}}{\partial r_{d}}\right|_{\mathrm{eq}}=\frac{r_{d}^{\mathrm{eq}}}{r_{2}^{\mathrm{eq}}}+\frac{m_{\mathrm{O}}}{m_{\mathrm{O}}+m_{\mathrm{N}}} \frac{r_{v}^{\mathrm{eq}}}{r_{2}^{\mathrm{eq}}} \cos \theta_{\mathrm{eq}}, \\
& B=\left.\frac{\partial r_{2}}{\partial r_{v}}\right|_{\mathrm{eq}}=\left(\frac{m_{\mathrm{O}}}{m_{\mathrm{O}}+m_{\mathrm{N}}}\right)^{2} \frac{r_{v}^{\mathrm{eq}}}{r_{2}^{\mathrm{eq}}}+\frac{m_{\mathrm{O}}}{m_{\mathrm{O}}+m_{\mathrm{N}}} \cos \theta_{\mathrm{eq}} \frac{r_{d}^{\mathrm{eq}}}{r_{2}^{\mathrm{eq}}} \\
& C=\left.\frac{\partial r_{3}}{\partial r_{d}}\right|_{\mathrm{eq}}=\frac{r_{d}^{\mathrm{eq}}}{r_{3}^{\mathrm{eq}}}-\frac{m_{\mathrm{N}}}{m_{\mathrm{O}}+m_{\mathrm{N}}} \cos \theta_{\mathrm{eq}}+\frac{r_{v}^{\mathrm{eq}}}{r_{3}^{\mathrm{eq}}} . \\
& D=\left.\frac{\partial r_{3}}{\partial r_{v}}\right|_{\mathrm{eq}}=\left(\frac{m_{\mathrm{N}}}{m_{\mathrm{O}}+m_{\mathrm{N}}}\right)^{2} \frac{r_{v}^{\mathrm{eq}}}{r_{3}^{\mathrm{eq}}}-\cos \theta_{\mathrm{eq}} \frac{m_{\mathrm{N}}}{m_{\mathrm{O}}+m_{\mathrm{N}}} \frac{r_{d}^{\mathrm{eq}}}{r_{3}^{\mathrm{eq}}} .
\end{aligned}
$$

Substituting in Eq. (63) and simplifying we obtain

$$
\begin{aligned}
V\left(r_{v}, r_{d}\right)= & \left(r_{v}-r_{v}^{\mathrm{eq}}\right)^{2}\left(\frac{1}{2} K_{1}+\frac{1}{2} K_{2} B^{2}+\frac{1}{2} K_{3} D^{2}+K_{23} B D\right) \\
& +\left(r_{d}-r_{d}^{\mathrm{eq}}\right)^{2}\left(\frac{1}{2} K_{2} A^{2}+\frac{1}{2} K_{3} C^{2}+K_{23} A C\right) \\
& +\left(r_{d}-r_{d}^{\mathrm{eq}}\right)\left(r_{v}-r_{v}^{\mathrm{eq}}\right)\left(K_{2} A B+K_{3} C D\right. \\
& \left.+K_{23} A D+K_{23} B C\right) .
\end{aligned}
$$

Now, substituting the values of $r_{d}$ and $r_{v}$ from Eq. (62) into Eq. (A12) we get the dimensionless quadratic potential $V_{1}$ used in Eq. (61) as

$$
\begin{aligned}
V_{1}(x, y)= & \frac{1}{2} K_{11}\left(x-x^{\mathrm{eq}}\right)^{2}+\frac{1}{2} K_{22}\left(y-y^{\mathrm{eq}}\right)^{2} \\
& +K_{12}\left(x-x^{\mathrm{eq}}\right)\left(y-y^{\mathrm{eq}}\right),
\end{aligned}
$$

where

$$
\begin{aligned}
K_{11}= & \left(r_{0}^{2} \mu_{v}\right) \frac{r_{0}^{2} \mu_{v}}{\mu_{d}}\left(K_{2} A^{2}+K_{3} C^{2}+2 K_{23} A C\right), \\
K_{22}= & \left(r_{0}^{2} \mu_{v}\right) r_{0}^{2}\left(K_{1}+K_{2} B^{2}+K_{3} D^{2}+2 K_{23} B D\right), \\
K_{12}= & \left(r_{0}^{2} \mu_{v}\right) \frac{r_{0} \sqrt{\mu_{v}}}{\sqrt{\mu_{d}}} \\
& \times\left(K_{2} A B+K_{3} C D+K_{23} A D+K_{23} B C\right), \\
x^{\mathrm{eq}=} & \frac{r_{d}^{\mathrm{eq}} \sqrt{\mu_{d}}}{r_{0} \sqrt{\mu_{v}}},
\end{aligned}
$$

and

$$
y^{\mathrm{eq}}=\frac{r_{v}^{\mathrm{eq}}}{r_{0}} .
$$

${ }^{1}$ H. Heather and H. Metiu, J. Chem. Phys. 90, 6903 (1989).

${ }^{2}$ V. Engel and H. Metiu, J. Chem. Phys. 91, 1596 (1989).

${ }^{3}$ D. G. Imre and J. Zhang, Chem. Phys. 139, 89 (1989).

${ }^{4}$ D. Kosloff and R. Kosloff, J. Comput. Phys. 52, 35 (1983).

${ }^{5}$ A. Askar and A. S. Cakmak, J. Chem. Phys. 68, 2794 (1978).

${ }^{6}$ R. E. Mickens, Phys. Rev. A 39, 5508 (1989).

${ }^{7}$ M. D. Feit, J. A. Fleck Jr., and A. Steiger, J. Comput. Phys. 47, 412 (1982).

${ }^{8}$ H. Tal-Ezer and R. Kosloff, J. Chem. Phys. 81, 3967 (1984).

${ }^{9}$ D. Neuhauser and M. Baer, J. Phys. Chem. 93, 2872 (1989).

${ }^{10}$ H. Heather and H. Meitu, J. Chem. Phys. 86, 5009 (1987).

${ }^{11}$ R. Kosloff and D. Kosloff, J. Comput. Phys. 63, 363 (1986).

${ }^{12}$ C. Leforestier, Chem. Phys. 87, 214 (1984).

${ }^{13}$ V. Mohan and N. Sathyamurthy, Comput. Phys. Rep. 7, 213 (1988)

${ }^{14}$ R. B. Gerber, R. Kosloff, and M. Berman, Comput. Phys. Rep. 5, 59 (1986).

15 (a) D. Bohm, Phys. Rev. 85, 166 (1952); (b) 85, 180 (1952).

${ }^{16}$ J. H. Weiner and A. Askar, J. Chem. Phys. 54, 3534 (1971).

${ }^{17}$ A. Askar and J. H. Weiner, Am. J. Phys. 39, 1230 (1971).

${ }^{18}$ F. S. Mayor, A. Askar, and H. A. Rabitz (unpublished).

${ }^{19}$ J. H. Weiner and Y. Partom, Phys. Rev. 187, 1134 (1969).

${ }^{20}$ J. H. Weiner and A. Askar, J. Chem. Phys. 54, 1108 (1971).

${ }^{21}$ J. H. Weiner and Y. Partom, Phys. Rev. B 1, 1533 (1970).

${ }^{22}$ S. K. Ghosh and B. M. Deb, Phys. Rev. A 92, 1 (1982).

${ }^{23}$ B. M. Deb and P. K. Chattaraj, Phys. Rev. A 37, 4030 (1988).

${ }^{24}$ B. K. Dey and B. M. Deb, Int. J. Quantum Chem. 56, 707 (1995).

${ }^{25}$ G. Terlecki, N. Grun, and W. Scheid, J. Phys. B 17, 3717 (1984).

${ }^{26}$ P. Zemmerer, N. Grun, and W. Scheid, Phys. Lett. A 134, 57 (1988).

${ }^{27}$ F. M. M. Faisal and U. Schwengelbeck, Phys. Lett. A 207, 31 (1995).

${ }^{28}$ U. Schwengelbeck and F. M. M. Faisal, Phys. Lett. A 199, 281 (1995).

${ }^{29}$ M. Horbatsch and R. M. Dreizler, Z. Phys. A 300, 117 (1981).

${ }^{30}$ J. P. Boris, A. M. Landsberg, E. S. Oran, and J. H. Gardner, LCPFCT, A Flux Corrected Transport Algorithm for Solving Generalized Continuity Equation, NRL, Washington, 1993. 
${ }^{31}$ D. L. Book, C. Li, G. Patnaik, and F. F. Grinstein, J. Sci. Comput. 6, 323 (1991).

${ }^{32}$ C. R. DeVore, J. Comput. Phys. 92, 142 (1991).

${ }^{33}$ R. Löhner, K. Morgan, J. Peraire, and M. Vahdati, Int. J. Num. Math. Fluids 7, 1093 (1987).

${ }^{34}$ S. T. Zalesak, J. Comput. Phys. 31, 335 (1979).

${ }^{35}$ U. Manthe, H. D. Meyer, and L. S. Cederbaum, J. Chem. Phys. 97, 3199 (1992).

${ }^{36}$ U. Manthe, H. D. Meyer, and L. S. Cederbaum, J. Chem. Phys. 97, 9062 (1992).
${ }^{37}$ R. Schinke, M. Nonella, H. U. Suter, and J. R. Huber, J. Chem. Phys. 93, 1098 (1990).

${ }^{38}$ A. Untch, K. Weide, and R. Schinke, J. Chem. Phys. 95, 6496 (1991).

${ }^{39}$ R. N. R. Dixon, C. C. Marston, and G. G. Balint-Kurti, J. Chem. Phys. 93, 6520 (1990).

${ }^{40}$ K. Weide and R. Schinke, J. Chem. Phys. 90, 7150 (1989).

${ }^{41}$ V. Engel, J. Chem. Phys. 94, 16 (1991).

${ }^{42}$ K. C. Kulander, C. Cerjan, and A. E. Orel, J. Chem. Phys. 94, 2571 (1991). 